\title{
Wireless relay placement optimization in underground room and pillar mines
}

\author{
Arpan Halber*, Debashish Chakravarty \\ Department of Mining Engineering, IIT Kharagpur, WB 721302, India
}

Corresponding Author Email: research.halder.arpan@gmail.com

https://doi.org/10.18280/mmep.050203

Received: 19 April 2018

Accepted: 26 May 2018

\section{Keywords:}

graph theory, mining engineering, combinatorial optimization, wireless infrastructure, WSNs

\begin{abstract}
For the implementation of real-time underground monitoring, communication and tracking, the deployment of wireless communication backbone inside underground mines has got the attention of the researchers in the recent decade. For wireless system deployment in the permanent structure of underground mines, wired communication backbones are appropriate as permanent districts serve for a longer time. However, for temporary openings where service life is for few months or weeks, communication backbone with wireless relay nodes may come out to be more economical as wireless relay nodes give flexibility with deployment, rearrangement and easy retreat. In recent literature multiple approaches of optimal relay node placements are available. However, the choice of best approach for an application is dependent on the specific requirement and constraints of that particular application site. This paper, we discuss on necessary requirements and constraints of wireless relay placement planning in an underground room and pillar mines. Considering the requirements and constraints that are discussed, an optimization approach for wireless relay placement in an underground room and pillar mines using network theory has been proposed in this paper.
\end{abstract}

\section{INTRODUCTION}

Applications of WSNs is recently a research trend in mining industry [1-9]. For the implementation of WSN based solutions in underground mines a network backbone is necessary. Unlike most other domestic application sites of WSN deployment, there are some additional challenges of establishing wireless communication infrastructure in underground room and pillar mines. In room and pillar working districts signal waves cannot penetrate through the pillars, and the wave propagation characteristic is also different in case of underground mines due to uneven walls of underground openings [10-14]. This is why for reliable wireless communication within underground mines, preferred means of wireless communication is within line of sight and up to a limited distances only. More importantly, the layout of room and pillar districts are itself dynamic; it changes with advancing working faces. There are counted few underground mines that have full-fledged $\mathrm{Wi}-\mathrm{Fi}$ data connectivity infrastructure, those are Chelopech gold mine, Bulgaria; Totten mine Worthington, Ontario; Éléonore gold mines James Bay, Quebec etcetera. At this point, the practice of having digital infrastructure in underground mine is still in initial phases, this is why there is yet no established/standardized method for full-scale deployment of wireless connectivity in the working districts of underground mines.

Underground mining operation mainly involves two types of structures, permanent structures and temporary structures. Permanent structures are those that serve for a long time (generally full lifetime of mine). Entry to an underground mine (shaft, incline, decline, adit etcetera) and immediate drives and crosscuts from the entry are the permanent structure of a mine.
The other structures like room and pillar districts, stopes etcetera are temporary structures created for mineral exploitation only, and shall be abandoned (or backfilled depending on the mining method used) after a short time [1517].

Deployment of wired data connectivity infrastructure is economical for permanent structures as it shall serve throughout the mine life. However, for temporary structures, a sensible approach is to deploy wireless connectivity with wireless relaying devices; such that when mining operation is over, then retrieval of the deployed data connectivity infrastructure will be easier. And a wireless communication infrastructure constructed using wireless relay nodes takes less time to deploy and retreat compared to its wired counterpart. In addition wireless deployment also provides flexibility for easy shifting with dynamically advancing faces and changing worksite layout of underground mines.

De-pillaring districts of an underground room and pillar mines are a common example of temporary structures in underground mines. In room and pillar method galleries are cut through the mineralized zone leaving the square pillars to support the hang-wall [15-17], and after the complete development the pillars are also excavated leaving a small fraction behind. Depending on the level of mechanization it takes time of weeks to months. For coal mines, this times within with work in the temporary district has to be completed is also constrained by the limitation of the incubation period. This is why in general this development and retreat at the time of de-pillaring is a fast process. With the advancement of mining extraction faces, the Regions of Interests (ROIs henceforth) where we require wireless coverage (for monitoring and tracking application) also change. In this situation establishing a data communication backbone in 
temporary districts is more convenient with wireless relay nodes. The network infrastructure deployment with wireless relay deployment help in getting rid operations like of wiring, removal and rearrangement of wiring during the retreat and shifting the network.

In this paper, we propose a method for planning the optimal wireless relay node placement through the rock/coal confined galleries of room and pillar district with a mathematical approach using network theory. In our approach, we first model the problem as a graph problem incorporating the constraints and the objectives. Then we've solved the formulated problem using Genetic Algorithm (GA) as the optimizer of this formulation.

In the following sections of paper, we've first elaborated on Regions of Interests (ROIs) in a room and pillar district where require having wireless coverage for purposes like tracking, communication, monitoring and other IoT aided applications. In subsequent section of this paper we have presented our proposed approach in details and a testing of this method on a stipulated room and pillar panel. Then respectively the results, discussion and conclusion relating to this work.

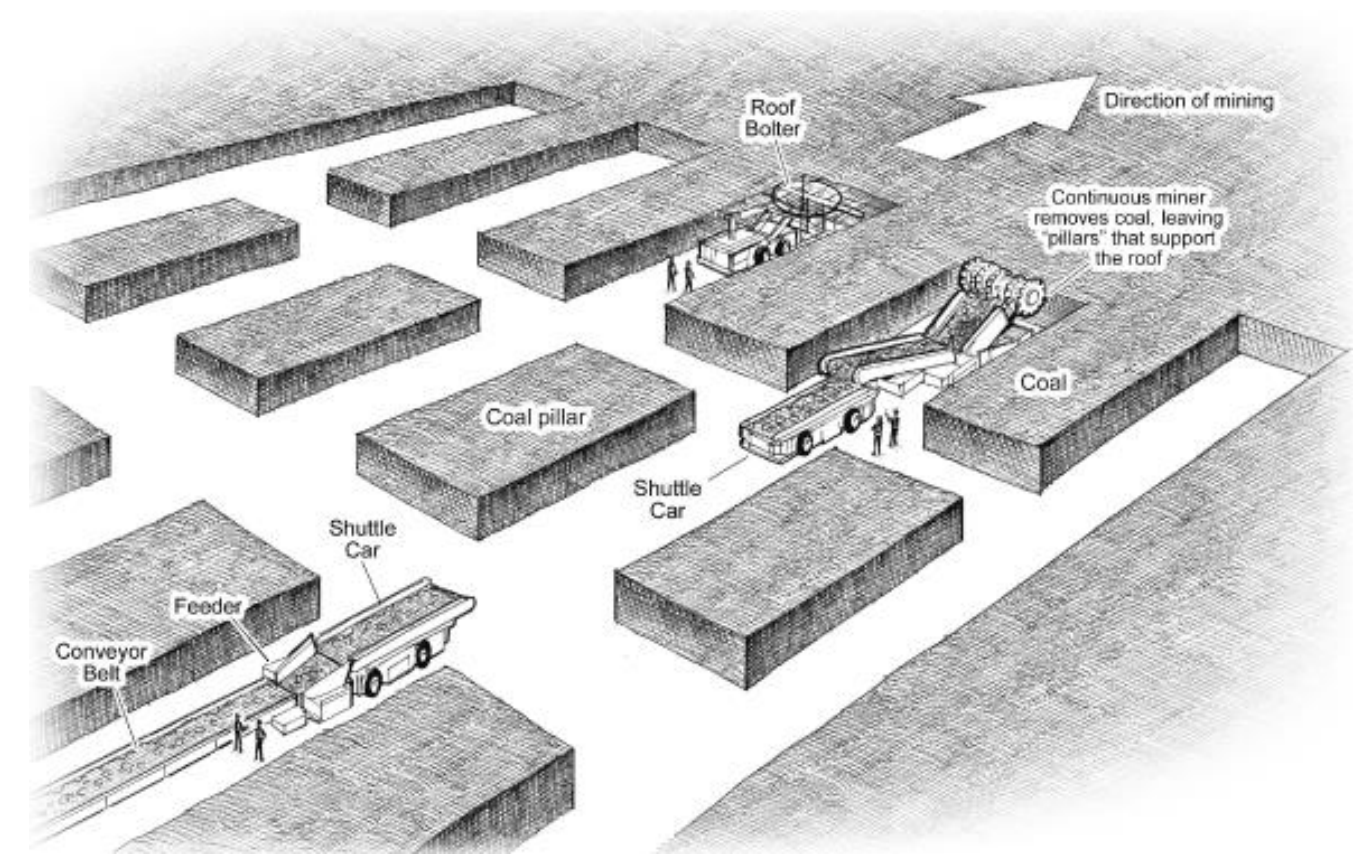

Figure 1. An illustration of room and pillar mining method. (Source: US Securities and Exchange Commission)

\section{REGIONS OF INTERESTS IN A ROOM AND PILLAR MINING DISTRICT}

One of the primary region of interest where we need wireless coverage within a mining district for monitoring and tracking is working faces, and the most taken routes along with men and material pass during the operation. Then there are key places in the district where real-time monitoring of gas concentration, rock mass parameters etcetera is required. For example, in Figure 6 shows a diagonal depillaring pattern, where in bottom right pillar the extraction is under process, during removal of material in an underground opening, the stress around the strata is re-distributed [16-18] this is why around the immediate extraction zones it is desired to have roof strata monitoring sensor devices. For monitoring of gas constrictions and temperature in the regions of interests is another concern especially in coal mines. For remote monitoring of such parameters generally sensor nodes are deployed. In fact for monitoring of such less time critical parameters such as gas concentration and temperature the technique similar to [19] where a mobile robot mounted with necessary sensors has been used to monitor less time critical parameters also have potential to be used in monitoring of gases and temperature in underground mine districts. The main point here is that the places which needs regular monitoring should get wireless data coverage.

While deploying wireless communication infrastructure, this has to be made sure that all the regions of interests are in coverage of the network. During the excavation in a district, the working faces and the route along which men and material move also change, this is why Regions of Interest (ROIs) change in the course of advancing excavation faces. Providing wireless coverage to all the ROIs is in general one of the primary objectives of network planning and optimization algorithms for underground mines [1,20].

The strategy for mathematical representation of ROIs in our formulation is explained further in the section 5.1 of this paper.

\section{CHALLENGES OF RELAY NODE PLACEMENT IN UNDERGROUND MINES}

The wireless relay placement algorithms that are designed for surface applications such as buildings are not suitable for application in underground room and pillar district as the rock/coal pillars are impermeable for radio waves (unlike building walls). Literature also report that wave propagation patterns inside underground mine is different from surface. Which is why for relay node placement planning in underground mines, a separate radio propagation modeling approaches is necessary to successfully predict radio signal propagation strength at the position where next relay node shall be placed. For that a relay node placement algorithm for underground mines should also take case of the radio wave 
propagation phenomenon within underground galleries. There have been several research approaches for coming up with a proper radio wave propagation model for underground mines $[10-13,21]$.

Next uniqueness of wireless relay placement problem in mines is the mine's layout that is with multiple number of galleries and junction. Which makes this problem different and more complex than relay placement problem in domestic structures. Another important point that needs a mention that in reality always the room and pillar districts layouts are not ordered and symmetric like of this examples in Figure 1, Figure 3 and Figure 6 . The Figure 2 shows such an asymmetric room and pillar plan. A method for relay node placement optimization in underground mines should preferably be able to implement such complex mine networks as well.

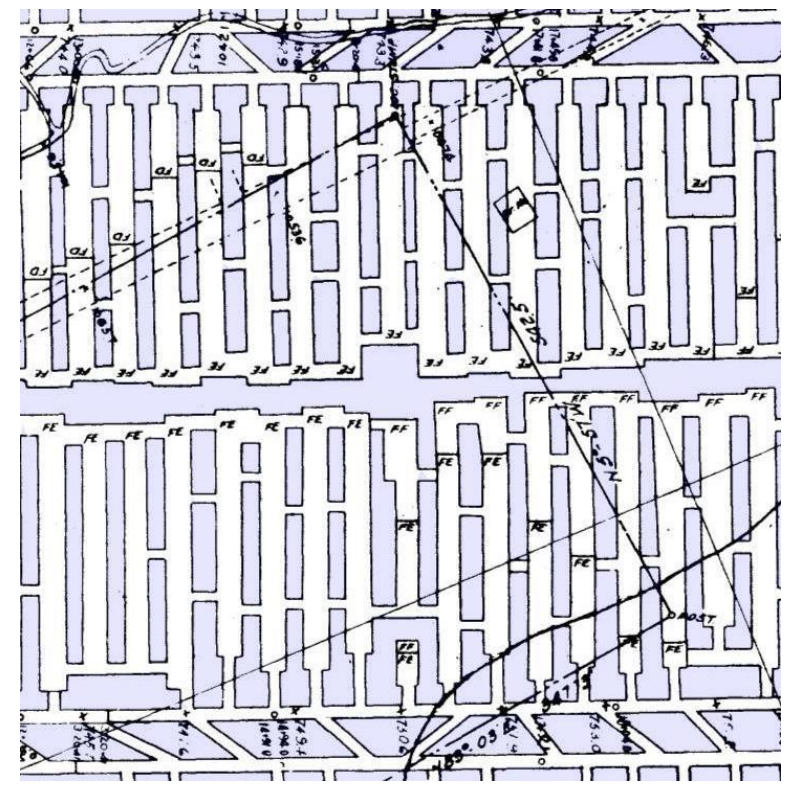

Figure 2. Example of an asymmetric room and pillar plan (Source: Pennsylvania Mine Map Atlas)

In addition, with advancing and retreating excavation faces of room and pillar mines the layout of mine change with advancement and retreat of excavation faces, thus the method for relay node placement problem also have to consider the dynamic nature of mine layout. This kind of characteristics are not common in case of surface wireless relay placement application. In this thesis [1] the author has addressed the problems by first establishing a tool that can predict the propagation and coverage of wireless signals within room and pillar mining district. Next for optimal placement of wireless nodes developed a heuristic based algorithm that that is suitable for optimal placement of wireless mesh network within underground room and pillar district. The work that is been presented in this paper is related to this second research gap of developing suitable method for optimal deployment of wireless relay node placement in underground room and pillar mines.

The relay placement method optimization method presented in this paper takes suitable measured to address the above challenges, which is discussed in Section 5 of this article.

\section{RELATED LITERATURE}

A wide range of both primary and secondary literature was surveyed to identify approaches used in several others fields and in the field of the mining industry as well. The first category of literature that relates this work are existing optimization approaches for solving this NP-hard relay placement problem [22-27]. Among them, the journal article [25] proposes a generalized approach for looking at constrained wireless relay placement problem as a graph problem. The network relay and cluster heard placement problem is concerned with several multi objective criteria, one of the important among those criteria is coverage and reliable connectivity among the nodes $[27,28]$. In mining industry application related literature, one of such work in modelling of wireless mesh relay optimization problem for underground coal mines is the doctoral thesis [1], where the thesis work in one of the chapters proposed modeling this wireless relay node placement problem in room and pillar mine as discreet optimization problem and explained the challenges, vastness of search space of solving the problem.

\subsection{Summary of relay placement algorithms}

Wireless relay node placement optimization is an NP-Hard problem. Depending on the type of applications and the constraints, relay placement problem is formulated in different approaches. However, the underlying algorithm for solving the formulation is either of exhaustive search, heuristic-based techniques or meta-heuristic based techniques. Different application sites have their specific constraints because of that reason, different variations in algorithms incorporating such case-specific constraints and complexities are designed by the researchers working in this domain.

The algorithms discussed in recent literature and literature from recent past can be seen in following sub-categories depending on its objectives.

(i) Algorithms trying to minimize the number of relay nodes to minimize the network cost [29].

The algorithms designed to achieve a fully connected network or a two-tiered network [25, 30-31].

Algorithms for ensuring reliability and fault tolerance, latency etcetera of the designed networks. Algorithms deal with case-specific constraints, for example, lifespan in case of battery powered relay nodes.

In general, depending on the application requirement algorithms often deal with multiple objectives.

Authors in [29], one of the work from the year 2005, proposed an approach to model wireless node placement problem with the minimum set covering problem, putting the constraints such that the total network cost is minimized while restrictions regarding coverage \& connectivity are satisfied. The authors in [30], an article from the year 2006, have proposed formulation of an algorithm that computes optimal number and optimal positions of wireless relay nodes in the playing in an open field in such a way that each sensor node can communicate with at least one RN, and the network of RNs is wholly connected with each other. These objective authors have achieved by means of discretizing the potential places of relay placement in the field by drawing grid lines. Authors in [32], an article from 2008 have proposed a method that uses the concept of Euclidean Steiner tree problem in the problem formulation and computes the optimal number and optimal positions of wireless relay nodes to maintain global connectivity restricting the locations of the relay nodes. The authors in [31] also have formulated the relay node placement 
problem based on Steiner tree minimization problem. Authors $[25,33]$ have formulated a generalized graph theory based model for WSN deployment planning that addresses the relay node placement optimization as well.

Now below are some relay node placement strategies that used meta-heuristic techniques to solve the relay node placement problems. The authors in [34] have discussed on three different types of coverage problems; simple, $k$-coverage and $Q$-coverage. Authors have proposed first a coverage formation algorithm which is followed by a cover optimization phase to create an optimized cover set. For connectivity problem, an $M$-connected optimized algorithm is proposed. In the optimization phase, it adds the remaining nodes one by one to the optimized cover set and checks for all possible $M$ connected subsets at each new addition. Thus the overall complexity of the algorithm becomes high. The authors in [35] proposed a GA based approach to deploy the sensor nodes for providing the maximum coverage in the network. In [28] the authors presented formulation to find the various locations to place a minimum number of relay nodes in such a manner that the nodes provide full coverage to the area and individual sensor nodes are connected with the network. And they have solved the formulation using a genetic algorithm. Fitness value of a chromosome is the number of sensor nodes required to provide the necessary connectivity and coverage. Another important aspect of their work is custom crossover and mutation function, which they found leading better result than the conventional crossover approaches. The authors in [23] solved the relay placement problem as using a genetic algorithm with a formulation that approximates the problem as a combinatory optimization problem; they too used customized crossover function for their operation.

This subsection roughly summarizes the algorithmic approaches taken by recent and past researchers in dealing with relay node placement problem.

\section{PROPOSED METHOD}

The roadway network of a room and pillar mine can be presented as a graph network. Where each roadway junction in the mine plan is treated as the nodes of the graph and the roadways joining the junctions are the node linking edges of the graph network representation. Figure 3 illustrates a sample room and pillar panel where its junction nodes are marked as 1 to 35 ; here in this case these 35 numbered points are potential place for relay node placement in this mine panel. The junction nodes of a room and pillar plan are most suitable candidate place for relay node placement $[1,36]$, because from the junctions nodes all the connected galleries get line of sight wireless coverage. Next the roadway sections of this panel then can be represented as the edges joining the nodes. For example roadway joining node 2 and 7 is presented by the "pair set' of nodes $(2,7)$.

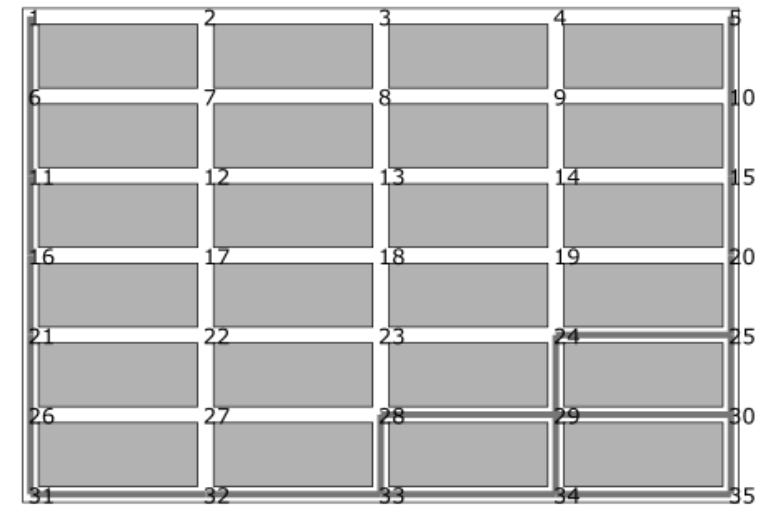

Figure 3. A sample room and pillar mine with numbered junction nodes

Table 1. Notations used in the article

SETs

\begin{tabular}{|c|c|}
\hline$R N$ & Set of all potential candidates for placements of Relay Nodes \\
\hline$R O A D W A Y$ & $\begin{array}{l}\text { Set of two adjacent nodes pair of the modelled mine panel parenting to a roadway section in the map of } \\
\text { the application site. }(u, v) \mid u \in R N, v \in R N .\end{array}$ \\
\hline$R O I$ & $\begin{array}{c}\text { Region of Interest ROI are those gallery sections which are to be given wireless coverage. } R O I \subseteq \\
\text { ROADWAYS }\end{array}$ \\
\hline \multicolumn{2}{|r|}{ Variables and Predicates } \\
\hline Linked $(a, b)$ & $\begin{array}{l}\text { A predicate that returns true if position 'a' and 'b' }(a \in R N, b \in R N) \text { are such that position 'a' and 'b' } \\
\text { are in wireless coverage range of each other. } \\
\text { In this formulation a simplified line of site based propagation model is considered where the predicate } \\
\text { returns true if position 'a' and 'b' are in the line of sight of each other. And distance between these two } \\
\text { nodes are less than reliable communication distance } R_{d} \text {. }\end{array}$ \\
\hline Connected $(G)$ & Predicate that returns true if Graph 'G' is a connected graph. \\
\hline Coverage $(G, I)$ & $\begin{array}{c}\text { A predicate that returns true if } I \in R O I \text { gets wireless coverage for the wireless network represented by } \\
\text { Graph } \mathrm{G} \text {. } \\
\begin{array}{c}\text { For, } I=(u, v) \text { output is true if }[\exists x \in V(G) \mid L(x, u) \wedge L(x, v)] \text {.This Lemma (1) holds true mostly for the } \\
\text { current approach with bord and pillar mining districts. }\end{array}\end{array}$ \\
\hline UG & $\begin{array}{l}\text { Is a Graph that represents a network whose nodes are all potential candidate of relay node placement in } \\
\text { the targeted mine district and edges are all feasible connections. Such that } \mathrm{V}(\mathrm{UG})=\mathrm{RN} \text { and } E(U G)= \\
\qquad\left[(a, b) \in R N^{2} \mid \operatorname{Linked}(a, b)\right]\end{array}$ \\
\hline \multicolumn{2}{|r|}{ Constants } \\
\hline $\mathcal{R}_{\mathrm{d}}$ & Distance to which wireless communication between relay nodes can be established reliably. \\
\hline$C_{n}$ & $\begin{array}{l}\text { A constant that represents the cost of each node deployment, it is not necessarily the actual cost but a } \\
\text { proportionate numerical value that is used as a representation of the cost of each wireless relay node. }\end{array}$ \\
\hline$P T_{R O I}$ & Value of penalty to fitness score in case an ROI is not covered by the solution. \\
\hline$P$ & alty to the fitness scores in case the solution fed to fitness function is a disconnected graph. \\
\hline
\end{tabular}


Now, we consider all the gallery junction points to be the candidate positions for relay node placement in the layout. And all those candidate positions are henceforth represented as the element of set 'RN'. All the gallery sections in mine layout are elements of set 'ROADWAY' and are represented as edges joining the respective nodes from set RN thus the set ROADWAY $\subseteq R N^{2}$. Each roadway section is represented as edges joining the respective roadway junction nodes from set ' $\mathrm{RN}$ '. In case of the underground room and pillar panels, all the gallery roadways are nearly straight so there is no scope of more than one link connecting the same pair of roadway junctions. Thus every roadway can uniquely be presented as pairs of $(u, v) \mid u \in R N, v \in R N$.

When we're planning the relay placement for underground room and pillar districts, the first objective we have is to give coverage to all our galleries where real-time tracking and monitoring is desired. The next priority is to minimize the number of hops from sensor end to gateway node. Finally, for ease of maintenance, we'd also like to achieve the objectives using the minimum number of nodes possible.

As the radio waves cannot propagate through coal pillars, in such case an acceptable means of establishing reliable communication links between relay nodes is by placing the adjacent nodes within line of sight (LOS) of each other. Such that there is only minor obstacles in the path of radio signal propagation between the nodes and seamless data transmission between the adjacent nodes is possible. Next factor is maximum permissible distance between the nodes within which reliable wireless transmission is possible. This distance up to which reliable communication is possible is dependent mainly on the type of hardware being used and radio wave path loss at the galleries. This distance $\mathcal{R}_{d}$ for a particular application has to be estimate through a suitable radio propagation model or via field experiments.

Next, to incorporate ROIs in this formulation the shaded region in Figure 3 are the regions of interest that is taken considering a diagonal de-pillaring pattern. In our formulation ROIs are the subset of all the roadways sections in the mining panel; thus for representing ROIs mathematically we consider a set ROI where $\boldsymbol{R} \boldsymbol{O I} \subseteq \mathrm{ROADWAY}$.

\subsection{Formulation}

This optimization has multiple objectives. The first objective is that all of its ROI's or the ROADWAYS that are of interest get wireless coverage. That is

$\forall I \in R O I \mid$ Coverage $(G, I)=$ True;

Next as constrain we need the network of relays to be a connected tree such that all the nodes are connected to the gateway node.

Connected $(G)=$ True;

Finally we'd like to achieve this with using minimal number of relay nodes, as having minimal number of relay nodes shall reduce maintenance requirements. Thus our objective is to minimize the number of nodes in the solution graph $\mathrm{G}$.

\section{Minimize $|V(G)|$}

A notable point here that search space for this problem is the power set $2^{V(U G)}$, that is all possible combinations of elements in $\mathrm{RN}$, this is the total search space among them some are feasible solution and rest are infeasible. So, for if 35 nodes present in $\mathrm{RN}$ or $V(U G)$ then the total set of configurations $2^{35}$, and searching this exhaustively means $2^{35}$ function calls; that a computationally heavy task. This is why some heuristic or some stochastic optimization technique has to be used for solving this problem.

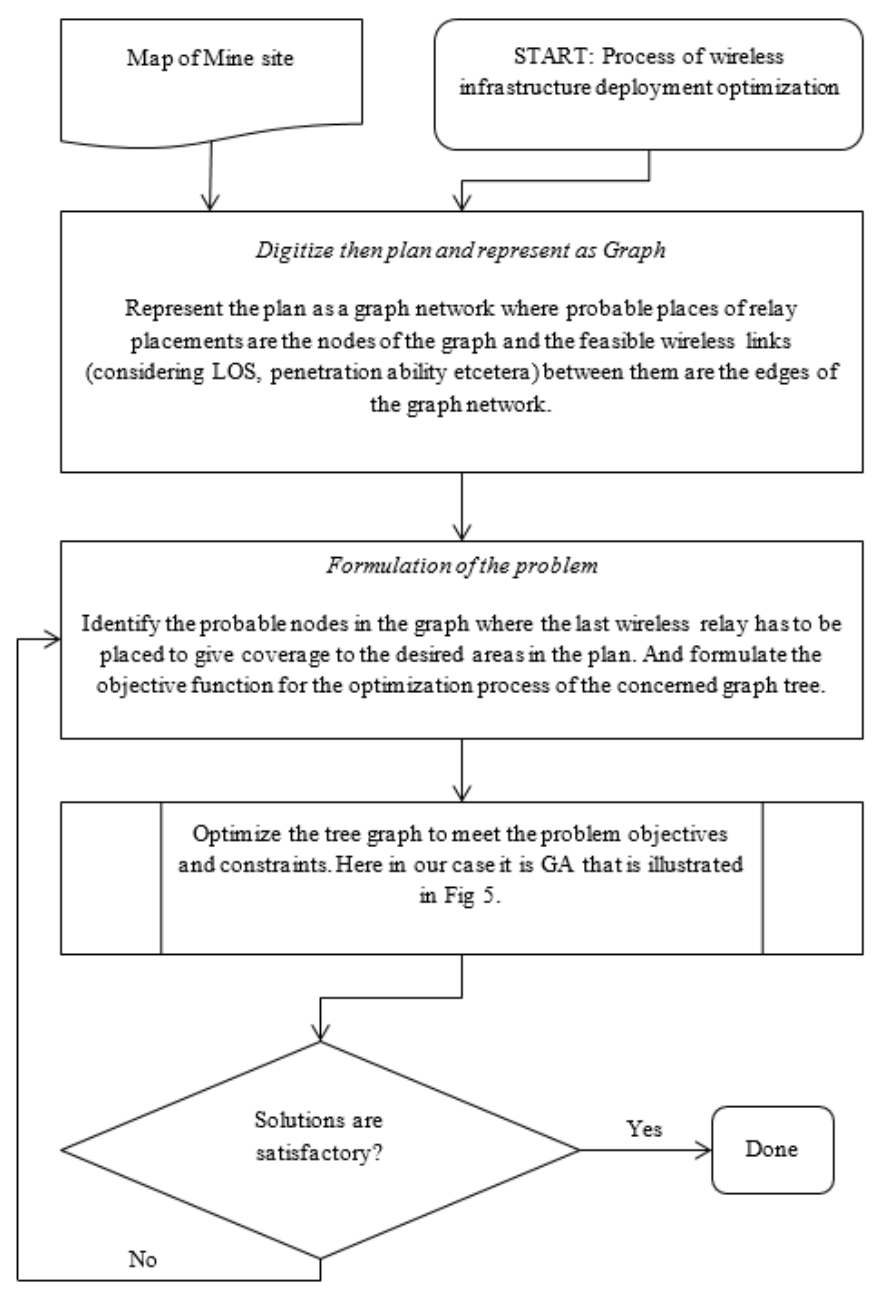

Figure 4. Flowchart of the proposed method

\subsection{Implementation of the proposed formulation}

This formulation was implemented using Genetic Algorithm. Where chromosome is a bit string that represents the state of all suitable candidate places for relay node placement (the Set RN) where if the particular bit is 1 that means a wireless relay is present in that candidate junction of room and pillar gallery.

Next, for checking the first constraint (Eq. 2) Depth First Search (DFS) traversing was used to check whether the graph $\mathrm{G}$ is connected, and a penalty is added $\mathrm{PT}_{\text {disconnected }}$ for violation of this constraint and rest of the computation is done removing the disjoined nodes from the consideration. Thereafter coverage criteria for each ROI is checked (Eq. 1) using the formulation explained in Lemma 1. And a moderately high penalty $P T_{R O I}$ is added for absence of coverage in each ROI.

Thus finally the Fitness value is:

Fitness $=|\mathrm{V}(\mathrm{G})| * \mathrm{C}_{\mathrm{n}}+\mathrm{PT}_{\text {disconnected }}$

$+\mathrm{PT}_{\mathrm{ROI}} *$ No. of uncovered ROI 
For the constants, we used the following values $C_{n}=1$; $P T_{R O I}=5 * C_{n} \quad ; \quad P T_{\text {disconnected }}=0.5 * C_{n} ; \quad$ in case Connected $(G)=$ True; $P T_{\text {disconnected }}=0$;.

At this point we've set this values out of intuition, in the future experiments analysis are to be done to get insights on what should be suitable values for this constants.

\section{Lemma 1}

For, $I=(u, v)$ output of Covarage $(G, I)$ is true if $[\exists x \in$ $V(G) \mid \operatorname{Linked}(x, u) \wedge \operatorname{Linked}(x, v)]$.

This Lemma shall hold true for all the room and pillar configuration modelled as a graph with this proposed approach. For understanding this lemma lets refer to Figure 3 in results section, if we take some $I=(7,8)$ in such case we see no such node in $\mathrm{V}(\mathrm{G})$ that is lined with both 7 and 8 simultaneously, (node are the dots shown in the Figure 3, V(G) $=\{1,2,12,14,20,22,23,24,25,27,29,33,34\})$. However, for $I=(9,15)$ both

Linked $(9,14)=$ True; $\operatorname{Linked}(4,14)=$ True.

This is why

$$
\begin{aligned}
& \text { Covarage }(G,(9,15))=\text { True; } \\
& \text { and Covarage }(G,(7,8))=\text { False; }
\end{aligned}
$$

for the case shown in Figure 3.

\section{EXPERIMENT ON A STIPULATED SCENARIO}

For initial testing of our approach, we take a 4 by 6 room and pillar district as shown in Figure 3 . Here the set of RN = $\{1,2, \ldots, 35\}$ and the node 1 is taken as Sink Node that is directly connected to the network gateway in this mesh network. The Region of Interests (ROIs) in a mining panel are those shaded galleries where real-time tracking and monitoring is desired. The shaded galleries as shown in Figure 3 have been taken as ROIs for this optimization problem.

In this problem we take $\mathcal{R}_{d}$ for the relay devices to be $60 \mathrm{~m}$, the pillar dimensions in Figure 3 is $50 \mathrm{~m}$ by $20 \mathrm{~m}$ and the gallery width $5 \mathrm{~m}$. Such configuration makes the relay node to be able to communicate only upto the next junction along the pillar length as the junction next to it is more distant than $\mathcal{R}_{d}$. Next, along the pillar width, the device can communicate upto two subsequent junctions in the line of sight. For example, (refer to Figure 3 ) a relay device in node 2 can cover upto node 1 and node 3 along pillar length and along the pillar width in can communicate to devices placed within the LOS along node 7 , node 12 only. Based on this rule of reach the adjacency metrics or the feasible link between the nodes is decided.

However, the case we're stipulating for simulating the algorithm's performance on an idealized case scenario has some limitations. As discussed earlier, the room and pillar gallery network for an underground mine can be as complex as the map shown in Figure 2. And the case we are considering in our simulation is the idealized map layout of rectangular grids. However, this limitation is not very significant, as the proposed optimization method deals mainly with the abstract graph representation of the network. Thus, variation in physical properties of the mine map from the grid-like case like the one simulated here will not be of much concern as long as the gallery and the connectivity constraints are modelled correctly, while developing the graph representation of the mine gallery network.

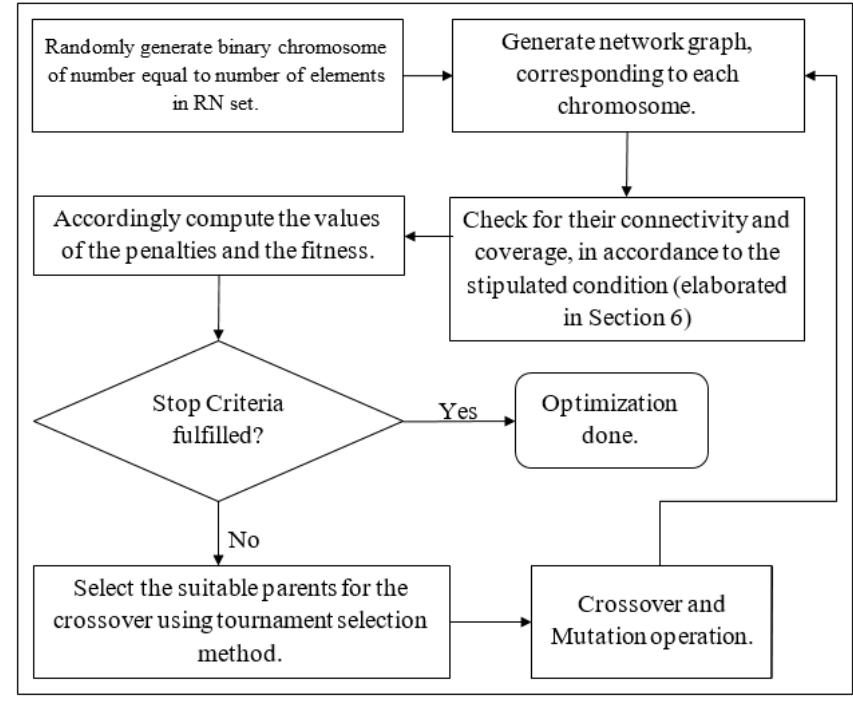

Figure 5. Flowchart of the GA implementation for this formulation

Thereafter optimization was run with 200 population size of binary chromosomes created randomly. With the other parameters as follows: mutation rate 0.05 , mutation function uniform, crossover faction 0.8 , crossover function scattered, selection function tournament with tournament size 4 and the elite count was set as 10 . Stopping criteria or the termination condition for this optimization was such that if there is no improvement in best fitness value for consecutive 50 generations, it assumes that solution has converged to global optimal and the GA is thus terminated.

\section{RESULTS}

For the above case, we found the best solution meeting the formulated constraints are the solutions using a minimum of 11 relay nodes; one of such solutions presented in Figure 6. Within the GA population, there were other feasible solutions as well using more nodes.

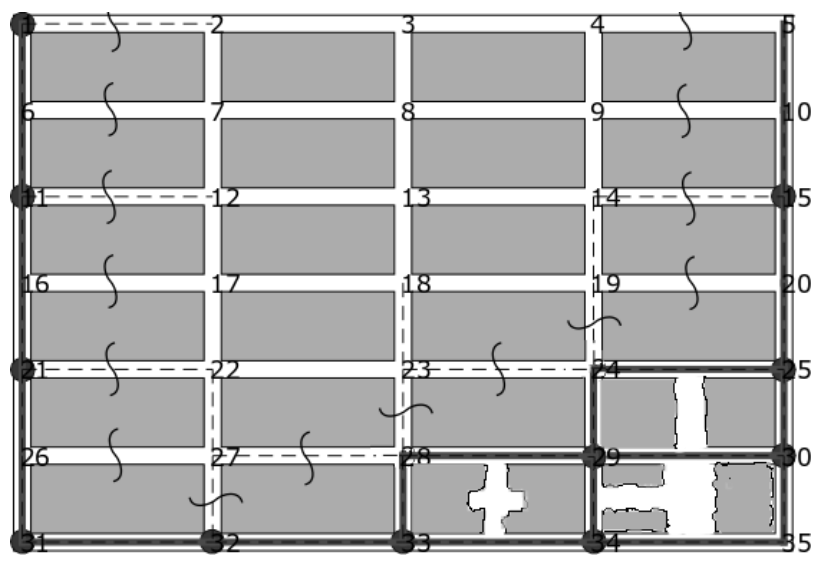

Figure 6. An optimal result covering all the ROIs found using this proposed approach; the black dots correspond to the wireless relay nodes

In Figure 6, the dashed lines show the regions which are under wireless coverage with the node in its vicinity. The tide $(\sim)$ like signs in figure shows brattice curtains that are used in room and pillar mines to channel the ventilated air to working 
districts [16], [37]. These brattice curtains are often made of polymers that don't offer much attenuation to radio waves thus the signals can penetrate through them. The shaded galleries as shown in the Figure 6 have been taken as ROIs considering it a layout of a diagonal de-pillaring operation. And the dashed line show the range of wireless coverage of respective relay nodes.

\section{PERFORMANCE ANALYSIS OF THIS OPTIMIZATION APPROACH}

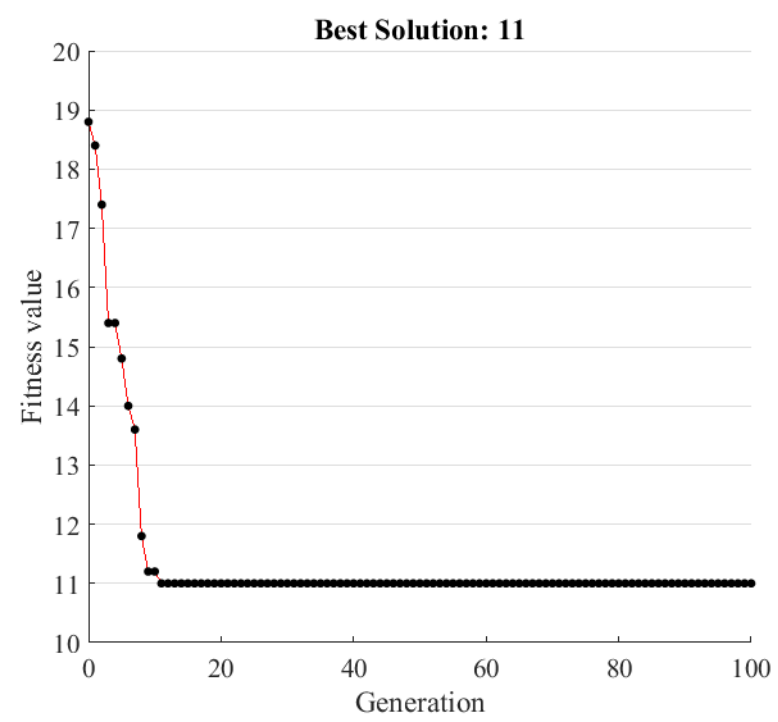

Figure 7. Convergence with population size 100

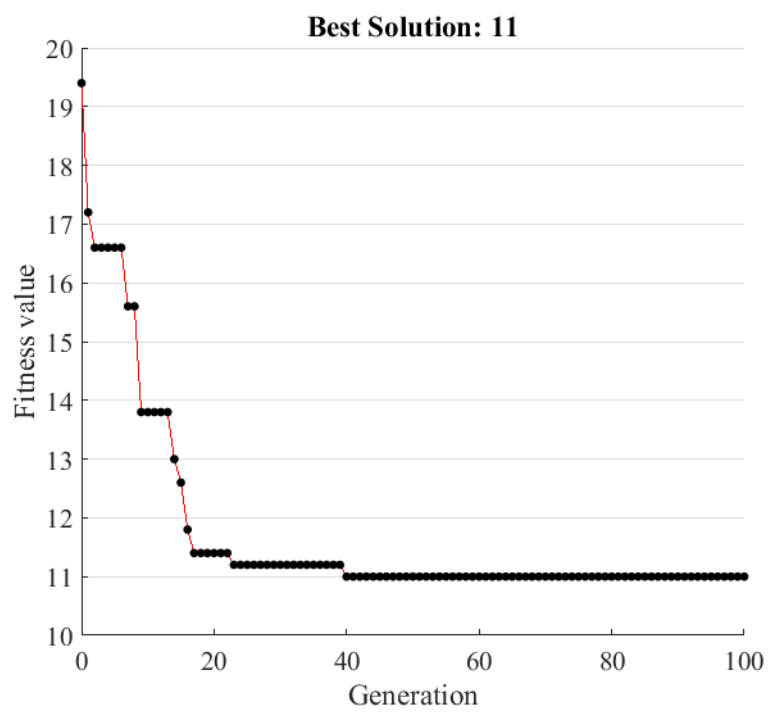

Figure 8. Convergence with population size 75

During optimization with the genetic algorithm, one of the primary criteria we observe is its convergence. A fast convergence means higher chances of reaching to some optimal solution with less number of iterations. For studying the convergence, we plot the generation vs the fitness value of the solution for four different population size $(25,50,75$ and $100)$, solving the same stipulated problem discussed in the Section 6. From these plots, we found when using 100 chromosomes as the population in the genetic algorithm it converges mostly within 20 generations. And performance in term of convergence decreases with a decrease in the number of the chromosome; as the algorithm takes more generation to converge to the solution. And we also observed, that in our experiment with population size 25 the algorithm couldn't reach the globally optimal solution. This algorithm performs reliably with higher population size only, we can conclude from this analysis.

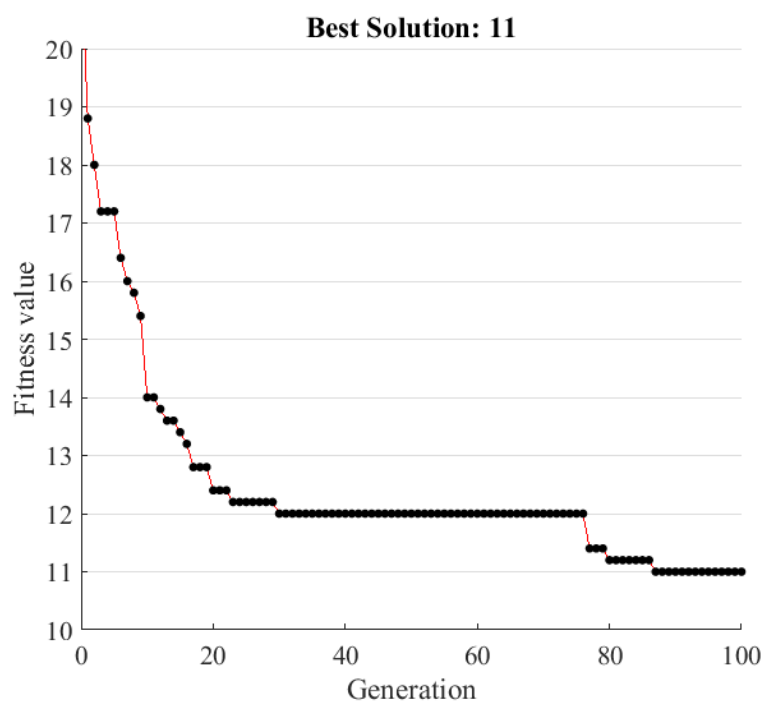

Figure 9. Convergence with population size 50

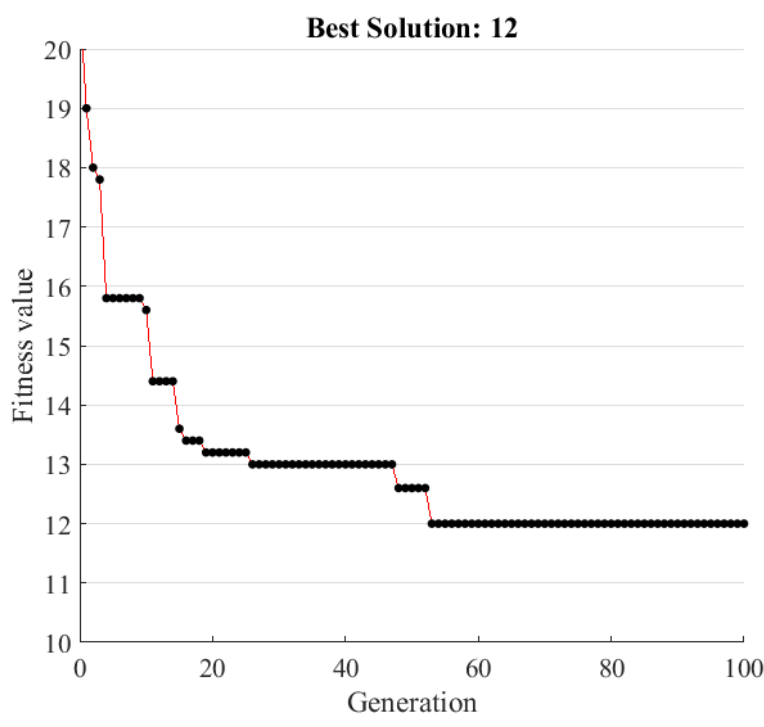

Figure 10. Convergence with population size 25

\section{DISCUSSION}

By running the GA optimizer for several times, we got the best solution reaching consistent solution with minimum of eleven relay nodes for this stipulated case. Where all the ROIs got the wireless coverage. From this observation, we assume that this optimization approach gives a globally optimal solution. For small number of pillars such planning can be done with intuition however, still using this optimizer is far more convenient than solving these relay node placement problem intuitively. As for larger maps human intuition without aid of some computer based optimization technique may lead to sub-optimal planning. More importantly, such 
deployment planning has to be done repeatedly for advancing and retreating faces of mining excavation. Compared to doing such repetitive planning operation with human intuition, a better option is automating this process using computers.

Currently we're optimizing the proposed formulation as the combinatorial optimization with a stochastic optimization tool that is genetic algorithm. Use of stochastic algorithm such as GA in this case helps getting optimal results for small application site such as small room and pillar district. However, if we try to optimize relay node placement in a large section of mine in such case optimization with heuristic based algorithm may work better in time complexity point of view. Though for limited number of pillars, currently proposed GA based approach is able to give optimal results within affordable time limits. Our proposed method has a limitation that it assumes reliable communication can be established only on the basis of line of sight and estimated reliable communication distance $R_{d}$. However, as per literature [11], [12], [14], [38] wave propagation patterns in underground mine galleries are similar to waveguides, and even in non-line of sight situation, around the corners, wireless coverage is there. In such case if we have a model that can accurately predict the coverage or a particular relay node, then we can model Linked $(a, b)$ predicate in a manner that it includes all the possible pairs of candidate positions between which wireless communication is possible.

\section{CONCLUSION}

This paper have tried to address the challenges of wireless relay node deployment planning within room and pillar mining districts by modelling the problem as a graph problem. The proposed approach in this article we've tested by formulating this optimization problem with this method for a stipulated room and pillar district and have solved it using a genetic algorithm to verify the consistency of this approach. The results we found with this approach seems feasible and optimal. Currently this method addresses the wireless relay optimization of a static snapshot; and for dealing with dynamic map change planning has to done one each instance. However, in future work it the dynamic changes of mine plan with face advancement can be formulated into the optimization so that whole optimization can be performed in one formulation.

\section{REFERENCES}

[1] Schafrik SJ. (2013). Underground Wireless Mesh Communication Infrastructure Design Prediction and Optimization (Doctoral) Virginia Polytechnic Institute and State University Retrieved from https://vtechworks.lib.vt.edu/handle/10919/19365

[2] Pablo Puñal Pereira. (2016). Efficient iot framework for industrial applications. PhD Thesis, Luleå University of Technology, Department of Computer Science.

[3] Moridi MA, Kawamura Y, Sharifzadeh M, Chanda EK, Wagner M, Jang H, Okawa H. (2015). Development of underground mine monitoring and communication system integrated ZigBee and GIS. International Journal of Mining Science and Technology 25(5): 811-818. https://doi.org/10.1016/j.ijmst.2015.07.017

[4] Vijayalakshmi SR, Muruganand S. (2016). Real Time Monitoring of Wireless Fire Detection Node. Procedia
Technology 24(Supplement C): 1113-1119. https://doi.org/10.1016/j.protcy.2016.05.244

[5] Chehri A, Fortier P, Tardif PM. (2009). UWB-based sensor networks for localization in mining environments. Ad Hoc Networks 7(5): 987-1000.

[6] Chehri A, Farjow W, Mouftah HT, Fernando X. (2011). Design of wireless sensor network for mine safety monitoring. 2011 24th Canadian Conference on Electrical and Computer Engineering (CCECE), pp. 001532-001535. https://doi.org/10.1109/CCECE.2011.6030722

[7] Henriques V, Malekian R. (2016). Mine safety system using wireless sensor network. IEEE Access 4: 35113521. https://doi.org/10.1109/ACCESS.2016.2581844

[8] Kiziroglou ME, Boyle DE, Yeatman EM, Cilliers JJ. (2017). Opportunities for sensing systems in mining. IEEE Transactions on Industrial Informatics 13(1): 278286. https://doi.org/10.1109/TII.2016.2636131

[9] Bhattacharjee S, Roy P, Ghosh S, Misra S, Obaidat MS. (2012). Wireless sensor network-based fire detection, alarming, monitoring and prevention system for Bordand-Pillar coal mines. Journal of Systems and Software 85(3):

$571-581$. https://doi.org/10.1016/j.jss.2011.09.015

[10] Jacksha R, Zhou C. (2016). Measurement of RF propagation around corners in underground mines and tunnels. Transactions of Society for Mining, Metallurgy, and Exploration, Inc. 340(1): 30-37.

[11] Forooshani AE, Bashir S, Michelson DG, Noghanian S. (2013). A survey of wireless communications and propagation modeling in underground mines. IEEE Communications Surveys Tutorials 15(4): 1524-1545. https://doi.org/10.1109/SURV.2013.031413.00130

[12] Boutin M, Benzakour A, Despins CL, Affes S. (2008). Radio wave characterization and modeling in underground mine tunnels. IEEE Transactions on Antennas and Propagation 56(2): 540-549. https://doi.org/10.1109/TAP.2007.913144

[13] Patri A, Nimaje DS. (2015). Radio frequency propagation model and fading of wireless signal at 2.4 $\mathrm{GHz}$ in an underground coal mine. Journal of the Southern African Institute of Mining and Metallurgy 115(7): 629-636. https://doi.org/10.17159/24119717/2015/V115N7A9

[14] Zhou C, Plass T, Jacksha R, Waynert JA. (2015). RF Propagation in Mines and Tunnels: Extensive measurements for vertically, horizontally, and crosspolarized signals in mines and tunnels. IEEE Antennas and Propagation Magazine 57(4): 88-102. https://doi.org/10.1109/MAP.2015.2453881

[15] Hartman HL, Mutmansky JM. (2002). Introductory Mining Engineering, John Wiley \& Sons.

[16] Christopher JB. (2013). Modern American Coal Mining: Methods and Applications, SME.

[17] Singh RD. (2014). Principles and Practices of Modern Coal Mining, New Age International.

[18] Dzimunya N, Radhe K, William C. (2018). Design and dimensioning of sublevel stoping for extraction of thin ore $(<12 \mathrm{~m})$ at very deep level: A case study of konkola copper mines $(\mathrm{kcm})$, Zambia. Mathematical Modelling of Engineering Problems 5(1): 27-32. https://doi.org/10.18280/mmep.050104

[19] Liu L, Shi Y, Long Y, Zhao J, Chen J, Cui Y. (2016). Greenhouse environment inspection vehicle control 
system design based on ZigBee. Mathematical Modelling of Engineering Problems 3(4): 184-190. https://doi.org/10.18280/mmep.030406

[20] Ding X, Shi L, Han J, Lu J. (2016). The study of crosslayer optimization for wireless rechargeable sensor networks implemented in coal mines. Sensors (Basel, Switzerland) 16(2). https://doi.org/10.3390/s16020171

[21] Liu X, Wang M, Wen J, Zhao Z. (2009). Transmission performance of $2.4 \mathrm{GHz}$ wireless sensor nodes when used in a working-face environment. Mining Science and Technology (China) 19(2): 185-188. https://doi.org/10.1016/S1674-5264(09)60035-1

[22] Lanza-Gutierrez JM, Gomez-Pulido JA. (2015). Assuming multiobjective metaheuristics to solve a threeobjective optimisation problem for Relay Node deployment in Wireless Sensor Networks. Applied Soft Computing 30(Supplement C): 675-687. https://doi.org/10.1016/j.asoc.2015.01.051

[23] George J, Sharma RM. (2016). Relay node placement in wireless sensor networks using modified genetic algorithm. 2016 2nd International Conference on Applied and Theoretical Computing and Communication. https://doi.org/10.1109/ICATCCT.2016.7912061

[24] Luo F. (2011). Relay Node Placement and Trajectory Computation of Mobile Data Collectors in Wireless Sensor NetworksUniversity of WindsorRetrieved from http://scholar.uwindsor.ca/etd/328

[25] Misra S, Hong SD, Xue G, Tang J. (2010). Constrained relay node placement in wireless sensor networks: Formulation and Approximations. IEEE/ACM Transactions on Networking 18(2): 434-447. https://doi.org/10.1109/TNET.2009.2033273

[26] Lanza-Gutierrez J, Gomez-Pulido JA, Vega-Rodríguez MA. (2013). A Trajectory Algorithm to Solve the Relay Node Placement Problem in Wireless Sensor Networks. https://doi.org/10.1007/978-3-642-45008-2_12

[27] Gupta SK, Kuila P, Jana PK. (2016). Genetic algorithm approach for $\mathrm{k}$-coverage and $\mathrm{m}$-connected node placement in target based wireless sensor networks. Computers \& Electrical Engineering 56: 544-556. https://doi.org/10.1016/j.compeleceng.2015.11.009

[28] Rebai M, Le berre M, Snoussi H, Hnaien F, Khoukhi L. (2015). Sensor deployment optimization methods to achieve both coverage and connectivity in wireless sensor networks. Computers \& Operations Research 59: 11-21. https://doi.org/10.1016/j.cor.2014.11.002

[29] Xu K, Wang Q, Hassanein H, Takahara G. (2005). Optimal wireless sensor networks (WSNs) deployment: minimum cost with lifetime constraint. IEEE International Conference on Wireless and Mobile Computing, Networking and Communications. https://doi.org/10.1109/WIMOB.2005.1512937

[30] Tang J, Hao B, Sen A. (2006). Relay node placement in large scale wireless sensor networks. Computer Communications 29(4): 490-501. https://doi.org/10.1016/j.comcom.2004.12.032

[31] Lee S, Younis M. (2012). Optimized relay node placement for connecting disjoint wireless sensor networks. Computer Networks 56(12): 2788-2804. https://doi.org/10.1016/j.comnet.2012.04.019

[32] Cheng X, Du D-Z, Wang L, Xu B. (2008). Relay sensor placement in wireless sensor networks. Wireless Networks 14(3): 347-355. https://doi.org/10.1007/s11276-006-0724-8

[33] Yang D, Misra S, Fang X, Xue G, Zhang J. (2012). Twotiered constrained relay node placement in wireless sensor networks: Computational complexity and efficient approximations. IEEE Transactions on Mobile Computing 11(8): 1399-1411. https://doi.org/10.1109/TMC.2011.126

[34] Mini S, Udgata SK, Sabat SL. (2014). Sensor deployment and scheduling for target coverage problem in wireless sensor networks. IEEE Sensors Journal 14(3): 636-644. https://doi.org/10.1109/JSEN.2013.2286332

[35] Yoon Y, Kim YH. (2013). An efficient genetic algorithm for maximum coverage deployment in wireless sensor networks. IEEE Transactions on Cybernetics 43(5): 1473-1483. https://doi.org/10.1109/TCYB.2013.2250955

[36] Griffin KR, Schafrik SJ, Karmis M. (2010). Designing and modeling wireless mesh communications in underground coal mines. 2010 SME Annual Meeting, Phonex, AZ, pp. 10-066

[37] McPherson MJ. (1993). Subsurface Ventilation and Environmental Engineering, Springer Netherlands, Dordrecht. https://doi.org/10.1007/978-94-011-1550-6

[38] Hrovat A, Kandus G, Javornik T. (2014). A survey of radio propagation modeling for tunnels. IEEE Communications Surveys Tutorials 16(2): 658-669. https://doi.org/10.1109/SURV.2013.091213.00175 